\title{
Isotropic Tensor Decomposition of Cartan's Curvature Tensor In Complex Finsler Manifolds
}

\author{
K. C. Petwal ${ }^{1}$ and Monika Sati ${ }^{2}$ \\ Department ${ }^{1,2}$ of Mathematics, \\ H.N.B. Garhwal University (A Central University) \\ S.R.T. Campus BadshahiThaul, Tehri Garhwal \\ Uttarakhand, India \\ Email:monikasati123@gmail.com
}

\begin{abstract}
In this paper our main purpose is to discuss some techniques of higher order decomposition of well-known Cartan's first curvature tensor $S_{i j k h}$. Moreover, we attempted to establish few significant results that may produce vital connections between Complex Finsler Manifold and Riemannian Christoffel Symbol (Curvature Tensor). Also, by adopting the techniques of decomposition, various cases and conditions have been developed.
\end{abstract}

Keywords: Finsler; Manifol; Riemannian; Christoffel; Isotropic; Cartan’s Curvature; Decomposition; Tensor

\section{INTRODUCTION}

A tensor which has the property that all its components are same in all the co-ordinate system is said to be isotropic tensor. Even if either the co-ordinate system or medium is rotated (by rotation we mean either rotation by 180 degree where the determinant of rotation matrix is 1 or complete rotation in which determinant of reflection matrix is -1), all the components of tensor remains same. Otherwise, tensor is anisotropic in nature.
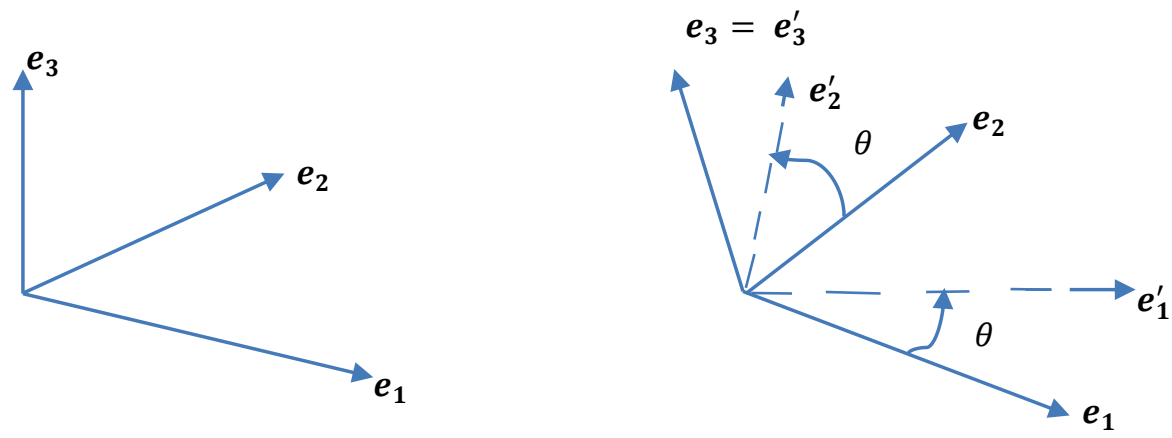

Figure I.1: The original Co-ordinate system I (solid lines) and the rotated system I' (dashed line).

A fourth order tensor can be considered as a conjunction of second order tensors. We know that the second order isotropic tensor is the identity tensor which is also symmetric. The possible conjunction of second order identity tensors are $\varphi_{k h}, \varphi_{i k}, \varphi_{j h}, \varphi_{i h}, \varphi_{j k}$. Therefore, we can write

$$
\begin{aligned}
a_{i j k h} & =\varphi_{i j} \varphi_{k h}, \\
b_{i j k h} & =\varphi_{i k} \varphi_{j h}, \\
c_{i j k h} & =\varphi_{i h} \varphi_{j k}
\end{aligned}
$$

and the given these three conjunctions of the general fourth order tensors are:

$$
I_{i j k h}=c_{1} \varphi_{i j} \varphi_{k h}+c_{2} \varphi_{i k} \varphi_{j h}+c_{3} \varphi_{i h} \varphi_{j k}
$$

Fourth order tensors have been used widely in the field of fine element method and computational mechanics in the last twenty years. Few important applications of them include tangent (elastic and elasto-plastic) moduli as well as damage tensors which play important role in formulation of evolution and constitutive equations. Due to absence of closed theory embracing many important aspects, the use of fourth order tensors remain complicated.

The fundamental idea of a Finsler manifold may be traced back to the famous lecture of Riemann: "Uber die hypothese, welche der Geometrie zugrunde liegen”. In this memoir of 1854 Riemann discusses various possibilities by means of which an n-dimensional manifold may be endowed with a metric, and pays particular attention to a metric defined by the positive square root of a positive definite quadratic differential form.

Thus the foundations of Riemannian geometry are laid; nevertheless. It is also suggested that the positive fourth root of a fourth order differential form might serve as a metric function. These functions have three properties in common: they are positive, homogeneous of the first 
degree in the differentials, and are also convex in the latter. It would seem natural, therefore, to introduce a further generalisation to the effect that the distance $d s$ between two neighbouring points represented by the coordinates $w^{i}$ and $w^{i}+d w^{i}$ be defined by some function $F\left(w^{i}, d w^{i}\right)$ in a complex Finsler manifold as:

$$
d s=F\left(w^{i}, d w^{i}\right) \forall(i=1,2, \ldots \ldots \ldots, n)
$$

Again, the theory took a new and unexpected turn in 1934 when E. Cartan published his tract on Finsler spaces. He showed that it was indeed possible to define connection coefficients and hence a covariant derivative such that the preservation of Ricci's lemma was ensured. On this basic, Cartan developed a theory of curvature and practically all subsequent investigations concerning the geometry of Finsler spaces were dominated by this approach. Several mathematicians expressed the opinion that the theory had thus attained its final form.

The above- mentioned theories make use of a certain device which basically involves the consideration of a space whose elements are not the points of the underlying manifold, but the line- elements of the latter, which form a $(2 n-1)$ dimensional variety. This facilitates the introduction of what Cartan calls the "Euclidean connection", which by means of certain postulates, may be derived uniquely from the fundamental metric function $F\left(w^{i}, d w^{i}\right)$. The method also depends on the introduction of a so-called "element of support", namely, that at each point a previously assigned direction must be given, which then serves as directional argument in all functions depending on direction as well as position. Thus, for instance, the length of a vector and the vector obtained from it by an infinitesimal parallel displacement depend on the arbitrary choice of the element of support. It is this device which led to the development of Finsler geometry in terms of direct generalisations of the methods of Riemannian geometry.

\subsection{Preliminaries}

Fundamental postulate of E. Cartan: The theory of E. Cartan which treats the Finsler manifolds from an entirely different point of view has played the most prominent role in the development of the Finsler Geometry. In this section, we shall take a brief look on Cartan's monograph in which he discussed his postulates by means of covariant derivatives.

In order to be able endows, the Finsler manifold $F_{n}^{(C)}$ with so-called "Euclidean connection" Cartan considered the manifold $X_{2 n-1}$ of the line element $\left(w, \dot{w}^{l}\right)$ which is $(2 n-1)$ dimensional, since only the ration of $\dot{w}^{l}$ are necessary define a direction in the tangent manifold $T_{n}\left(\dot{w}^{l}\right)$. The co-ordinates are referring to the centre of the line element $\left(w^{i}, \dot{w}^{l}\right)$. All quantities

$$
S_{i j k h}+S_{i k h j}+S_{i h j k}=0
$$

Proof: Let us assume that, these three quantities $\left(R_{i j k h}, S_{i j k h}, I_{i j k h}\right)$ are equivalent to each other

Let, $\quad S_{i j k h}=g_{i \alpha} S_{j k h}^{\alpha}$

$$
R_{i j k h} \sim S_{i j k h} \sim I_{i j k h}
$$

Also,

$$
S_{i j k h}=g_{i \alpha}\left[\frac{\partial}{\partial x^{k}}\left\{\begin{array}{c}
\alpha \\
j h
\end{array}\right\}-\frac{\partial}{\partial x^{h}}\left\{\begin{array}{c}
\alpha \\
k h
\end{array}\right\}+\left\{\begin{array}{c}
\beta \\
j h
\end{array}\right\}\left\{\begin{array}{c}
\alpha \\
\beta k
\end{array}\right\}-\left\{\begin{array}{c}
\beta \\
k j
\end{array}\right\}\left\{\begin{array}{c}
\alpha \\
\beta h
\end{array}\right\}\right]
$$

such as tensors are to be defined by means of the functions of line elements.

An analytic expression would come into existence, infinitesimal small change and becomes $\left(w^{i}+\right.$ $\left.d w^{i}, \dot{w}^{l}+d \dot{w}^{l}\right)$. This variation of $X^{i}$ will be represented by means of a covariant differential:

$D X^{i}=d X^{i}+C_{k h}^{i}(w, \dot{w}) X^{k} d \dot{w}^{h}+\Gamma_{k h}^{i}(w, \dot{w}) X^{k} d w^{h}$, where the coefficients $C_{k h}^{i}$ and $\Gamma_{k h}^{i}$ are the functions of element of support. equation.

$$
D X^{i}=0 \text { or } d X^{i}=-C_{k h}^{i} X^{k} d \dot{w}^{\imath}-\Gamma_{k h}^{i} X^{k} d w^{i},
$$

en the length of $X^{i}$ remains invariant.

rank identity tensor for isotropic: The fourth rank identity tensor I maps any second rank, symmetric ensor $\mathrm{U}$ to itself

$$
\mathrm{I}: \mathrm{U}=\mathrm{U}
$$

It is straight forward to work out the components of (accounting for both minor and major symmetries)

$$
I_{i j k h}=\frac{1}{2}\left(\varphi_{i k} \varphi_{j h}+\varphi_{i h} \varphi_{j k}\right)
$$

Properties of Riemann - Christoffel Tensors of first ind and fourth order:

$$
\begin{array}{ll}
\text { i. } & R_{j i k h}=-R_{i j k h} \\
\text { ii. } & R_{i j h k}=-R_{i j k h} \\
\text { iii. } & R_{k h i j}=R_{i j k h} \\
\text { iv. } & R_{i j k h}+R_{i k h j}+R_{i h j k}=0
\end{array}
$$

\section{TENSOR DECOMPOSITION OF CARTAN'S CURVATURE TENSOR IN COMPLEX FINSLER MANIFOLDS}

some techniques for isotropic tensor decompositions are able to reduce the dimensionality or to transform cartan's curvature tensor in Riemann enthe following techniques: Cartan's curvature tensor as the cyclic order Riemann curvature tensor) and $S_{i j k h}$ (fourth order Cartan's curvature tensor) behave like, Isotropic tensor $I_{i j k h}$. The cyclic properties of these quantities are given by 


$$
=g_{i \alpha} \frac{\partial}{\partial x^{k}}\left\{\begin{array}{c}
\alpha \\
j h
\end{array}\right\}-g_{i \alpha} \frac{\partial}{\partial x^{l}}\left\{\begin{array}{c}
\alpha \\
k h
\end{array}\right\}+g_{i \alpha}\left\{\begin{array}{c}
\beta \\
j h
\end{array}\right\}\left\{\begin{array}{c}
\alpha \\
\beta k
\end{array}\right\}-g_{i \alpha}\left\{\begin{array}{c}
\beta \\
k h
\end{array}\right\}\left\{\begin{array}{c}
\alpha \\
\beta j
\end{array}\right\}
$$

Or, $\quad g_{i \alpha} \frac{\partial}{\partial x^{k}}\left\{\begin{array}{c}\alpha \\ j h\end{array}\right\}=\frac{\partial}{\partial x^{k}}\left[g_{i \alpha}\left\{\begin{array}{c}\alpha \\ j h\end{array}\right\}\right]-\left\{\begin{array}{c}\alpha \\ j h\end{array}\right\} \frac{\partial g_{i \alpha}}{\partial x^{k}}=\frac{\partial[j h, i]}{\partial x^{k}}-\left\{\begin{array}{c}\alpha \\ j h\end{array}\right\} \frac{\partial g_{i \alpha}}{\partial x^{k}}$

Similarly for

By the formula

$$
g_{i \alpha} \frac{\partial}{\partial x^{h}}\left\{\begin{array}{c}
\alpha \\
k h
\end{array}\right\}=\frac{\partial[j k, h]}{\partial x^{h}}-\left\{\begin{array}{c}
\alpha \\
j k
\end{array}\right\} \frac{\partial g_{i \alpha}}{\partial x^{h}}
$$

Using (3.4) (3.5) in eq. (3.3) we get

$$
\begin{aligned}
S_{i j k h}= & \frac{\partial[j h, i]}{\partial x^{k}}-\left\{\begin{array}{c}
\alpha \\
j h
\end{array}\right\} \frac{\partial g_{i \alpha}}{\partial x^{k}}-\frac{\partial[j k, i]}{\partial x^{h}}+\left\{\begin{array}{c}
\alpha \\
j k
\end{array}\right\} \frac{\partial g_{i \alpha}}{\partial x^{h}}+g_{i \alpha}\left\{\begin{array}{c}
\beta \\
j h
\end{array}\right\}\left\{\begin{array}{c}
\alpha \\
\beta k
\end{array}\right\}-g_{i \alpha}\left\{\begin{array}{c}
\beta \\
j k
\end{array}\right\}\left\{\begin{array}{c}
\alpha \\
\beta k
\end{array}\right\} \\
= & \frac{\partial[j h, i]}{\partial x^{k}}-\left\{\begin{array}{c}
\alpha \\
j h
\end{array}\right\} \frac{\partial g_{i \alpha}}{\partial x^{k}}-\frac{\partial[j k, i]}{\partial x^{h}}+\left\{\begin{array}{c}
\alpha \\
j k
\end{array}\right\} \frac{\partial g_{i \alpha}}{\partial x^{h}}+g_{i \alpha}\left\{\begin{array}{c}
\beta \\
j h
\end{array}\right\}\left\{\begin{array}{c}
\alpha \\
\beta k
\end{array}\right\}-g_{i \alpha}\left\{\begin{array}{c}
\beta \\
j k
\end{array}\right\}\left\{\begin{array}{c}
\alpha \\
\beta h
\end{array}\right\} \\
= & \frac{\partial[j h, i]}{\partial x^{k}}-\frac{\partial[j k, i]}{\partial x^{h}}+\left\{\begin{array}{c}
\alpha \\
j k
\end{array}\right\} \frac{\partial g_{i \alpha}}{\partial x^{h}}-\left\{\begin{array}{c}
\alpha \\
j h
\end{array}\right\} \frac{\partial g_{i \alpha}}{\partial x^{k}}+g_{i \alpha}\left\{\begin{array}{c}
\beta \\
j h
\end{array}\right\}\left\{\begin{array}{c}
\alpha \\
\beta k
\end{array}\right\}-g_{i \alpha}\left\{\begin{array}{c}
\beta \\
j k
\end{array}\right\}\left\{\begin{array}{c}
\alpha \\
\beta h
\end{array}\right\} . \\
S_{i j k h}= & \frac{\partial[j h, i]}{\partial x^{k}}-\frac{\partial[j k, i]}{\partial x^{h}}+\left\{\begin{array}{c}
\alpha \\
j k
\end{array}\right\}([i h, \alpha]+[\alpha h, i]) \\
& -\left\{\begin{array}{c}
\alpha \\
j h
\end{array}\right\}([i k, \alpha]+[\alpha k, i])+\left\{\begin{array}{c}
\beta \\
j h
\end{array}\right\}[\beta k, i]-\left\{\begin{array}{c}
\beta \\
j k
\end{array}\right\}[\beta h, i] \\
S_{i j k h} \quad= & \frac{\partial[j h, i]}{\partial x^{k}}-\frac{\partial[j k, i]}{\partial x^{h}}+\left\{\begin{array}{c}
\alpha \\
j k
\end{array}\right\}[i h, \alpha]-\left\{\begin{array}{c}
\alpha \\
j h
\end{array}\right\}[i k, \alpha]
\end{aligned}
$$

It is also written as

$$
\left.\left.S_{i j k h} \quad=\left|\begin{array}{cc}
\frac{\partial}{\partial x^{k}} & \frac{\partial}{\partial x^{h}} \\
{[j k, i]} & {[j h, i]}
\end{array}\right|+\mid \begin{array}{cc}
\alpha \\
i k
\end{array}\right\} \begin{array}{c}
\alpha \\
i h
\end{array}\right\}
$$

But we know that

$$
\begin{array}{ll}
{[j h, i]} & =\frac{1}{2}\left[\frac{\partial g_{h i}}{\partial x^{k}}+\frac{\partial g_{j i}}{\partial x^{h}}-\frac{\partial g_{j h}}{\partial x^{i}}\right] \\
{[j k, i]} & =\frac{1}{2}\left[\frac{\partial g_{k i}}{\partial x^{j}}+\frac{\partial g_{j i}}{\partial x^{k}}-\frac{\partial g_{j k}}{\partial x^{i}}\right]
\end{array}
$$

Using (2.8) and (2.9), equation (2.6) implies

$$
S_{i j k h}=\frac{1}{2} \frac{\partial}{\partial x^{k}}\left[\frac{\partial g_{k i}}{\partial x^{j}}+\frac{\partial g_{j i}}{\partial x^{h}}-\frac{\partial g_{j k}}{\partial x^{i}}\right]-\frac{1}{2} \frac{\partial}{\partial x^{l}}\left[\frac{\partial g_{k i}}{\partial x^{j}}+\frac{\partial g_{j i}}{\partial x^{h}}-\frac{\partial g_{j k}}{\partial x^{i}}\right]+\left\{\begin{array}{c}
\alpha \\
j k
\end{array}\right\}[i h, \alpha]-\left\{\begin{array}{c}
\alpha \\
j h
\end{array}\right\}[i k . \alpha]
$$

Or,

$$
S_{i j k h}=\frac{1}{2}\left[\frac{\partial^{2} g_{i h}}{\partial x^{j} \partial x^{k}}+\frac{\partial^{2} g_{j k}}{\partial x^{h} \partial x^{i}}-\frac{\partial^{2} g_{i k}}{\partial x^{j} \partial x^{h}}-\frac{\partial^{2} g_{j h}}{\partial x^{i} \partial x^{k}}\right]+\left\{\begin{array}{c}
\alpha \\
j k
\end{array}\right\}[i h, \alpha]-\left\{\begin{array}{c}
\alpha \\
j h
\end{array}\right\}[i k, \alpha]
$$

Since, $\quad\left\{\begin{array}{c}\alpha \\ j k\end{array}\right\}=g^{\alpha \beta}[j k, \beta] \quad$ And $\quad\left\{\begin{array}{c}\alpha \\ j h\end{array}\right\}=g^{\alpha \beta}[j h, \beta]$

Therefore,

$$
S_{i j k h}=\frac{1}{2}\left[\frac{\partial^{2} g_{i h}}{\partial x^{j} \partial x^{k}}+\frac{\partial^{2} g_{j k}}{\partial x^{i} \partial x^{h}}-\frac{\partial^{2} g_{i k}}{\partial x^{j} \partial x^{h}}-\frac{\partial^{2} g_{j h}}{\partial x^{i} \partial x^{k}}\right]+g^{\alpha \beta}[j k, \beta][i h, \alpha]-g^{\alpha \beta}[j h, \beta][i k, \alpha],
$$

Similarly,

$$
\begin{aligned}
& S_{i k h j}=\frac{1}{2}\left[\frac{\partial^{2} g_{i j}}{\partial x^{k} \partial x^{h}}+\frac{\partial^{2} g_{k h}}{\partial x^{i} \partial x^{j}}-\frac{\partial^{2} g_{i h}}{\partial x^{k} \partial x^{j}}-\frac{\partial^{2} g_{k j}}{\partial x^{i} \partial x^{h}}\right]+g^{\alpha \beta}[k h, \beta][i j, \alpha]-g^{\alpha \beta}[k j, \beta][i h, \alpha], \\
& S_{i h j k}=\frac{1}{2}\left[\frac{\partial^{2} g_{i k}}{\partial x^{h} \partial x^{j}}+\frac{\partial^{2} g_{h j}}{\partial x^{i} \partial x^{k}}-\frac{\partial^{2} g_{i j}}{\partial x^{h} \partial x^{k}}-\frac{\partial^{2} g_{h k}}{\partial x^{i} \partial x^{j}}\right]+g^{\alpha \beta}[h j, \beta][i k, \alpha]-g^{\alpha \beta}[h k, \beta][i j, \alpha]
\end{aligned}
$$

Adding equations (2.11), (2.12) and (2.13), we get eq. (2.1) i.e.

This completes the proof of theorem.

$$
S_{i j k h}+S_{i k h j}+S_{i h j k}=0
$$

In order to make the significance of above expression the following cases are arises:

Case I: The total number of distinct non- vanishing components of Cartan tensor $S_{i j k h}$ with four distinct indices is $\frac{1}{12} n^{2}\left(n^{2}-1\right) \quad, \quad$ where $i, j, k, h=$ $1,2,3, \ldots \ldots \ldots, n$. Forn $=1$, the number of distinct non - vanishing components of Cartan's curvature tensor $S_{i j k h}=0$ i.e. scalar, hence after decomposition of fourth rank tensor we reduce it to zero rank tensor and curve seems to be a straight line.
Case II: For $n \geq 1$ or higher order components of Cartan's curvature tensor $S_{i j k h}$, the non- vanishing components seems to be a curve and total number of distinct non - vanishing components does not exceed $\frac{1}{12} n^{2}\left(n^{2}-1\right)$.

Remark: When $S_{i j k h}$ is the form $S_{i i i i}$ (i.e. $i=j=k=$ $h)$ then $S_{i i i i}$ has no any component.

Technique II: Using Finsler equation to decompose the Cartan's curvature tensor in symmetric and antisymmetric property of isotropic tensor.

Theorem 2.2. Under the decomposition of fourth order isotropic tensor $\left(I_{i j k h}\right)$, in the form of Cartan's 
curvature tensor $\left(S_{i j k h}\right)$, behave like symmetric and anti-symmetric tensor and the relation between them is

$$
I_{i j k h}=S_{i j k h}=\frac{1}{2}\left(\varphi_{i k} \varphi_{j h}+\varphi_{i h} \varphi_{j k}\right)+\frac{1}{2}\left(\varphi_{i k} \varphi_{j h}-\varphi_{i h} \varphi_{j k}\right)
$$

Proof: Making use the concept of covariant differentiation of the equation as:

$$
\left.D\left\|S_{i j k h}\right\|=D\left[\frac{1}{2} \text { (Symmetric Part }\right)+\frac{1}{2}(\text { Anti-Symmetric Part })\right]
$$

For the feasibility, we write the covariant differentiation given by equation (2.14) with respect to $w^{i}$ as below

$$
D\left\|S_{i j k h}\right\|=\frac{1}{2} D\left[\left\|\varphi_{i k} \varphi_{j h}\right\|+\left\|\varphi_{i h} \varphi_{j k}\right\|\right]+\frac{1}{2} D\left[\left\|\varphi_{i k} \varphi_{j h}\right\|-\left\|\varphi_{i h} \varphi_{j k}\right\|\right]
$$

Using by part covariant differentiation in equation (2.15)

$$
\begin{aligned}
D\left[\left\|\varphi_{i k} \varphi_{j h}\right\|+\|\right. & \left.\varphi_{i h} \varphi_{j k} \|\right] \\
& =\frac{1}{2}\left[\varphi _ { i k } \left\{d\left|\varphi_{j h}\right|-\left|\varphi_{r h}\right|\left(\left|C_{j s}^{r}\right|(w, \dot{w}) d \dot{w}^{s}+\left|\Gamma_{j s}^{r}\right|(w, \dot{w}) d w^{s}\right)\right.\right. \\
& \left.-\left|\varphi_{j r}\right|\left(\left|C_{h s}^{r}\right|(w, \dot{w}) d \dot{w}^{s}+\left|\Gamma_{j s}^{r}\right|(w, \dot{w}) d w^{s}\right)\right\} \\
& +\varphi_{j h}\left\{d\left|\varphi_{i k}\right|-\left|\varphi_{r k}\right|\left(\left|C_{i s}^{r}\right|(w, \dot{w}) d w^{s}+\left|\Gamma_{l s}^{r}\right|(w, \dot{w}) d w^{s}\right)\right. \\
& \left.-\left|\varphi_{i r}\right|\left(\left|C_{k s}^{r}\right|(w, \dot{w}) d \dot{w}+\left|\Gamma_{k s}^{r}\right|(w, \dot{w}) d w^{s}\right)\right\} \\
& +\varphi_{i h}\left\{d\left|\varphi_{j k}\right|-\left|\varphi_{r k}\right|\left(\left|C_{j s}^{r}\right|(w, \dot{w}) d \dot{w}^{s}+\left|\Gamma_{j s}^{r}\right|(w, \dot{w}) d w^{s}\right)\right. \\
& \left.-\left|\varphi_{j r}\right|\left(\left|C_{k s}^{r}\right|(w, \dot{w}) d \dot{w}^{s}+\left|\Gamma_{k s}^{r}\right|(w, \dot{w}) d w^{s}\right)\right\} \\
& +\varphi_{j k}\left\{d\left|\varphi_{i h}\right|-\left|\varphi_{r h}\right|\left(\left|C_{i s}^{r}\right|(w, \dot{w}) d \dot{w}^{s}+\left|\Gamma_{i s}^{r}\right|(w, \dot{w}) d w^{s}\right)\right. \\
& \left.\left.-\left|\varphi_{i r}\right|\left(\left|C_{h s}^{r}\right|(w, \dot{w}) d \dot{w}^{s}+\left|\Gamma_{h s}^{r}\right|(w, \dot{w}) d w^{s}\right)\right\}\right]
\end{aligned}
$$

And

$$
\begin{aligned}
D\left[\left\|\varphi_{i k} \varphi_{j h}\right\|-\|\right. & \left.\varphi_{i h} \varphi_{j k} \|\right] \\
& =\frac{1}{2}\left[\varphi _ { i k } \left\{d\left|\varphi_{j h}\right|-\left|\varphi_{r h}\right|\left(\left|C_{j s}^{r}\right|(w, \dot{w}) d \dot{w}^{s}+\left|\Gamma_{j s}^{r}\right|(w, \dot{w}) d w^{s}\right)\right.\right. \\
& \left.-\left|\varphi_{j r}\right|\left(\left|C_{h s}^{r}\right|(w, \dot{w}) d \dot{w}^{s}+\left|\Gamma_{j s}^{r}\right|(w, \dot{w}) d w^{s}\right)\right\} \\
& +\varphi_{j h}\left\{d\left|\varphi_{i k}\right|-\left|\varphi_{r k}\right|\left(\left|C_{i s}^{r}\right|(w, \dot{w}) d w^{s}+\left|\Gamma_{l s}^{r}\right|(w, \dot{w}) d w^{s}\right)\right. \\
& \left.-\left|\varphi_{i r}\right|\left(\left|C_{k s}^{r}\right|(w, \dot{w}) d \dot{w}+\left|\Gamma_{k s}^{r}\right|(w, \dot{w}) d w^{s}\right)\right\} \\
& -\varphi_{i h}\left\{d\left|\varphi_{j k}\right|-\left|\varphi_{r k}\right|\left(\left|C_{j s}^{r}\right|(w, \dot{w}) d \dot{w}^{s}+\left|\Gamma_{j s}^{r}\right|(w, \dot{w}) d w^{s}\right)\right. \\
& \left.-\left|\varphi_{j r}\right|\left(\left|C_{k s}^{r}\right|(w, \dot{w}) d \dot{w}^{s}+\left|\Gamma_{k s}^{r}\right|(w, \dot{w}) d w^{s}\right)\right\} \\
& -\varphi_{j k}\left\{d\left|\varphi_{i h}\right|-\left|\varphi_{r h}\right|\left(\left|C_{i s}^{r}\right|(w, \dot{w}) d \dot{w}^{s}+\left|\Gamma_{i s}^{r}\right|(w, \dot{w}) d w^{s}\right)\right. \\
& \left.\left.-\left|\varphi_{i r}\right|\left(\left|C_{h s}^{r}\right|(w, \dot{w}) d \dot{w}^{s}+\left|\Gamma_{h s}^{r}\right|(w, \dot{w}) d w^{s}\right)\right\}\right]
\end{aligned}
$$

Adding equations(2.16) and(2.17), we get

$$
\begin{gathered}
D\left\|S_{i j k h}\right\|=D\left[\left\|\varphi_{i k} \varphi_{j h}\right\|+\left\|\varphi_{i h} \varphi_{j k}\right\|\right]+D\left[\left\|\varphi_{i k} \varphi_{j h}\right\|-\left\|\varphi_{i h} \varphi_{j k}\right\|\right] \\
=\left\|\varphi_{i k} \varphi_{j h}\right\|
\end{gathered}
$$

This is the symmetric part of isotropic tensor.

Hence after decomposition of isotropic tensor in the form of Cartan's curvature tensor behave like symmetric tensor and the relation (2.14) is satisfied. Also we define the following:

Definition 2.1. $D\left\|S_{i j k h}\right\|=I_{i j k h}$ is the isotropic symmetric tensor, where

$$
I_{i j k h}=\frac{1}{2}\left(\varphi_{i k} \varphi_{j h}+\varphi_{i h} \varphi_{j k}\right) .
$$

Therefore, Cartan curvature tensor $S_{i j k h}$ is symmetric as: isotropic tensor $I_{i j k h}$ is symmetric. Hence $S_{i j k h}$ is covariant symmetric tensor of rank four called fundamental covariant tensor.

\section{CONCLUSION}

In this paper we have relevant a theory of higher order tensor decomposition. The theory entitled to solve several suitable problems in the decomposition of higher order tensor, which are still chase the letter. The decomposition of higher order rank tensor to lower order rank tensor shows by the process of cyclic rotation of curve to make a straight line.
By adopting the process of covariant differentiation for the symmetric and anti-symmetric part of isotropic tensor, we have developed an expression which most probably predicts some complicated relations among the components of isotropic tensor and various tensorial and non-tensorial quantities. The relation (2.18) is vitally important as it describes direct or indirect co-relation between components of complex isotropic tensor and Christoffel second kind bracket symbol, Cartan tensor, scalar curvature, Riemann metric tensor as well as a third order tensor $C_{r s}^{p}(w, \dot{w})$ etc.

\section{REFERENCES}

[1] H. Rund. "The differential geometry of Finsler spaces". Springer- Verlag, 1959.

[2] H. Rund. "The curvature theory of directiondependent connections on complex manifolds". Tensor (N.S.), 24, 189-205, 1972.

[3] Nazrul Islam. "Tensors and their application". new age International (P) limited, Publishers, 2006. 
[4] K.C. Petwal and S. Kumar. "Techniques for the decomposition of Cartan's curvature tensor in complex Finsler manifolds". Albannian journal of mathematics, volume 5, no.2,67-86, 2011.

[5] K. Yano. "Differential geometry on complex and almost complex spaces".Pergamon Press, 1965.

[6] L. Hesselink, Y. Levy and Y. Levin. "The topology of symmetric, second order $3 \mathrm{~d}$ tensor fields". IEEE Transactions on visualization and Computer Graphics, 3(1) X,1-11, 2011.

[7] P.G. Bergmann. "Introduction to the theory of relativity $\left(5^{\text {th }}\right.$ Edition)". prentice hall of India Private limited, New Delhi, 1992.

[8] Mikhail Itskov. "On the theory of fourth-order tensors and their applications in Computational Mechanics". Elsevier, Computer methods in applied mechanics and engineering, 189, 419438, 2000.

[9] T. Hazan, S. Polka and A. Shashua. "Workshop on tensor decomposition and applications" CIRM, Marseille, France 2005.

[10] Ioan Bucataru. "A setting for higher order differential equation fields and higher order Lagrange and Finsler spaces". The journal of geometric mechanics, September 2013.

[11] Maher Moakher. "Fourth- order Cartesian tensors: old and new facts, notions and applications". The Quarterly Journal of mechanics and applied mathematics, January 2008.

[12] Chandrasekhar Jog. "A concise proof of the representation theorem for fourth - order isotropic tensors". Journal of elasticity, November 2006.

[13] By Vincent Monchiet and Guy Bonnet. "Inversion of higher order isotropic tensors with minor symmetries and solution of higher order heterogeneity problems". Proc. R. Soc. A 467, 314-332, 2011.

[14] M.E. Gurtin. "A short proof of the representation theorem for isotropic". linear stress-strain relations, J. Elasticity, 4 , 243245,1974.

[15] J. Betten. "Invariants of fourth-order tensors, in: J.P. Boehler (Ed.)" Applications of tensor functions in solid mechanics, CISM courses and lectures No. 292, Wein, , pp. 171-186, 1987. 